\title{
Trombose venosa cerebral: a relevância da neuroimagem
}

\author{
Cerebral venous thrombosis: the neuroimaging relevance
}

Trombosis venosa cerebral: la relevancia de la neuroimagen

Luana Fernandes da Silva Oliveira Castro ${ }^{1 *}$, Lorena Sipauba Pitanga ${ }^{1}$, Maria Júlia Ribeiro da Costa $^{1}$, Geovanna Godinho Santos ${ }^{1}$, Gabriela Almeida Lôbo¹, Fernanda Rodrigues Mangabeira

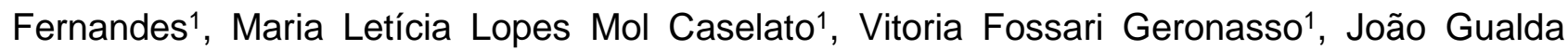
Garrido Trajano', Jobe Petter ${ }^{1}$.

\section{RESUMO}

Objetivo: Analisar a importância da neuroimagem no diagnóstico da trombose venosa cerebral. Revisão bibliográfica: A Trombose Venosa Cerebral (TVC) possui uma evolução inespecífica, a grande maioria dos pacientes apresenta cefaleia como manifestação inicial, podendo ou não estar associado a sintomas focais. Diante dos inúmeros sinais e sintomas que podem estar presentes, o diagnóstico clínico se torna improvável, pois além da sintomatologia inespecífica, a TVC pode mimetizar inúmeras doenças neurológicas. Assim, a Tomografia Computadorizada (TC) e a Ressonância Magnética (RM), associadas ou não a venografia, assumem um protagonismo indubitável no diagnóstico da trombose das veias e seios cerebrais. Em geral, o primeiro exame de imagem solicitado é a TC, contudo, para uma avaliação mais minuciosai e precisa do sistema cerebral a RM é preferível. Apesar de ser uma condição grave, o diagnóstico precoce e a intervenção terapêutica definem um bom prognóstico ao paciente, sendo crucial, portanto, a precisão e a velocidade na realização do diagnóstico, prevenindo assim, complicações e desfechos fatais. Considerações finais: A análise dos artigos estudados, evidenciaram a relevância dos métodos de neuroimagem como medidas indispensáveis para o diagnóstico definitivo de trombose venosa cerebral.

Palavras-chave: Trombose cerebral, Neuroimagem, Sistema nervoso central.

\begin{abstract}
Objective: To analyze the importance of neuroimaging in the diagnosis of cerebral venous thrombosis. Bibliographic review: The Cerebral Venous Thrombosis (CVT) has a nonspecific evolution, most patients referred headache as the first manifestation, which can be associated or not with focal symptoms. In addition to the countless signs and symptoms that can be found, the clinical diagnosis becomes unlikely, therefore, besides the nonspecific symptoms, the CVT can mimic innumerous neurological diseases. Thus, Computed Tomography (CT) and Magnetic Resonance Imaging (MRI), associated or not with venography, play an undoubted role in the diagnosis of thrombosis of cerebral veins and sinuses. In general, the first imaging exam requested is CT, however, for a more thorough and accurate assessment of the brain system, MRI is preferable. Despite being a severe condition, an earlier diagnosis and therapeutic intervention define a favorable prognosis, whereby the accuracy and speed in reaching the correct diagnosis are crucial, thus preventing complications and fatal outcomes. Final considerations: The analysis of the articles studied, showed the relevance of neuroimaging methods as indispensable measures for the definitive diagnosis of cerebral venous thrombosis.
\end{abstract}

Keywords: Cerebral thrombosis, Neuroimaging, Central nervous system.

${ }^{1}$ Centro Universitário do Planalto Central Apparecido dos Santos (UNICEPLAC), Brasília - DF.

*E-mail: luanafernandes2000@gmail.com 


\section{RESUMEN}

Objetivo: Analizar la importancia de la neuroimagen en el diagnóstico de trombosis venosa cerebral. Revisión bibliográfica: La trombosis venosa cerebral (TVC) tiene una evolución inespecífica, la mayoría de los pacientes presentan cefalea como manifestación inicial, que puede estar asociada o no a síntomas focales. En vista de los numerosos signos y síntomas que pueden estar presentes, el diagnóstico clínico se vuelve improbable, porque además de los síntomas inespecíficos, puede imitar numerosas enfermedades neurológicas. Así, la Tomografía Computada (TC) y la Resonancia Magnética (RM), asociada o no a la venografía, asumen un papel indudable en el diagnóstico de trombosis de las venas y senos del cerebro. En general, el primer examen por imágenes solicitado es la TC, sin embargo, para una evaluación más completa y precisa del sistema cerebral, es preferible la RM. A pesar de ser una enfermedad grave, el diagnóstico precoz y la intervención terapéutica definen un mejor pronóstico, por lo que la precisión y la rapidez en el diagnóstico son cruciales para evitar complicaciones y desenlaces fatales. Consideraciones finales: El análisis de los artículos estudiados mostró la relevancia de los métodos de neuroimagen como medidas indispensables para el diagnóstico definitivo de trombosis venosa cerebral.

Palabras clave: Trombosis cerebral, Neuroimagen, Sistema nervioso central.

\section{INTRODUÇÃO}

A Trombose Venosa Cerebral (TVC) é definida como uma doença cerebrovascular que caracteriza obstruções ocasionadas por trombos em seios ou veias cerebrais, determinando acometimento superficial ou profundo. É considerada uma doença rara, correspondendo a cerca de $0,5 \%$ a $1 \%$ de todos os eventos cerebrovasculares, sendo a maioria em pacientes com menos de 50 anos. Além disso, é a principal causa de Acidentes Vasculares Encefálicos (AVE) em pacientes jovens, sobretudo em mulheres, com idade média entre 25 e 40 anos. A TVC é um diagnóstico diferencial que deve ser considerado em todos os pacientes com hipertensão intracraniana isolada, encefalopatia, síndrome focal e crises convulsivas de etiologias desconhecidas (BALIERO LG, et al., 2020; ARAÚJO DS, 2017; GHONEIM A, et al., 2020; DMYTRIW AA, et al., 2018).

O grupo de risco predominantemente feminino é justificado pela forte associação da TVC à gravidez e ao puerpério, visto que o estado pró-trombótico tem um aumento de até seis vezes o risco de TVC. Portanto, além do uso de anticoncepcionais orais, uma história prévia ou familiar de trombose venosa profunda também são importantes fatores de risco que devem ser minuciosamente investigados durante a anamnese. Por outro lado, dentre os fatores de risco mais frequentes na população acima de 65 anos, trombofilia genética/adquirida, malignidade e distúrbios hematológicos, como policitemia, podem ser apontados (HERNÁNDEZ JLM, et al., 2019; OLIVEIRA GHR, et al., 2018; ZUURBIER SM, et al., 2018).

As causas de TVC são as mesmas de trombose originadas em outras regiões do corpo. Dessa forma, os fatores de risco estão associados com a clássica tríade de Virchow para trombogênese: hipercoagulabilidade, lesão endotelial e estase sanguínea. Geralmente a causa é multifatorial, isso indica que mesmo que um fator seja identificado como o causador da TVC, outras causas devem ser pesquisadas (ARAÚJO DS, 2017).

No que concerne ao diagnóstico da TVC, a equipe médica se depara com um impasse muito importante que é o diagnóstico tardio devido à ausência de sinais patognomônicos, bem como a apresentação muito semelhante a inúmeras doenças neurológica. Assim, por vezes o diagnóstico tardio pode variar entre 4 a 7 dias após o início dos sintomas, sendo um grande desafio identificar através das imagens radiológicas os estágios da TVC, que pode levar ao atraso para implementação de medidas terapêuticas e piorar o prognóstico (WU X, et al., 2021; GHONEIM A, et al., 2020; LONG B, et al., 2017).

As manifestações clínicas estão diretamente relacionadas com a localização do trombo, da duração da doença e das alterações do parênquima cerebral associadas à TVC. De maneira geral, podem ser categorizadas em: hipertensão intracraniana, disfunção cerebral focal, encefalopatia e convulsões com ou 
sem déficits neurológicos focais. Dessa forma, os exames de imagem são fundamentais no diagnóstico definitivo da doença. Atualmente, a Ressonância Magnética (RM), Tomografia Computadorizada (TC) e Angioressonância (ARM) são os exames de imagem com maiores recomendações no diagnóstico da TVC de acordo com a American Heart Association (AHA) e a European Federation of Neurological Societies (LV B, et al., 2021; ARAÚJO DS, 2017).

Apesar de grave, a TVC possui tratamento e cura. O diagnóstico em tempo hábil destaca-se como fator determinante no processo de cura do paciente. Dessa forma, a taxa de mortalidade pode variar entre $5 \%$ a $30 \%$, a depender da ausência de sinais e sintomas e diagnóstico tardio ou negligenciado. São frequentes variáveis preditivas de mortalidade: coma, alteração do estado de consciência, TVC profunda, hemorragia intracerebral direita, lesão na fossa posterior e agravamento de sinais neurológicos focais (ARAÚJO DS, 2017; OLIVEIRA GHR, et al., 2018).

O objetivo deste estudo foi revisar na literatura científica artigos, estudos e publicações atuais que discorressem sobre o papel da imagenologia e destacassem a relevância do diagnóstico por neuroimagem na Trombose Venosa Cerebral (TVC).

\section{REVISÃO BIBLIOGRÁFICA}

Em condições fisiológicas, cerca de 70-80\% do volume sanguíneo do cérebro se distribuem nas estruturas venosas cerebrais. Os seios venosos durais são responsáveis por drenar o sangue das veias cerebrais diretamente para as veias jugulares internas, além de colaborarem com a absorção de Líquor Cefalorraquidiano (LCR). Ademais, os seios venosos durais são canais sanguíneos localizados entre as camadas periosteal e meníngea da dura-máter, anastomosados e comumente fenestrados (ARAÚJO DS, 2017; GHONEIM A, et al., 2020).

Anatomicamente, os seios venosos durais são divididos em dois grupos: póstero-superiores e ânteroinferiores. O grupo póstero-superior envolve as veias maiores, compostas pelo seio sagital superior, seio sagital inferior, seio reto, a confluência dos seios (tórcula de Herófilo), seios transversais e sigmoides. Por outro lado, o grupo ântero-inferior é composto pelos seios petrosos superior e inferior, seio cavernoso e seio esfenoparietal (GHONEIM A, et al., 2020).

O sistema venoso cerebral pode ser subdividido em dois sistemas: superficial e profundo. $O$ sistema superficial drena a região do córtex e substância branca subcortical e é composto pelo seio sagital superior, veias corticais, seios transversos, seios sigmoides e seios cavernosos. O sistema profundo é formado pelas veias cerebrais internas, veias basais de Rosenthal, veia de Galeno e seio reto (ARAÚJO DS, 2017).

O sistema superficial ainda pode ser subdividido em três sistemas de drenagem: o grupo dorsomedial (drena para o seio sagital superior e seio reto), o grupo lateroventral (drena para os seios laterais - seios transversos e seios sigmoides) e o grupo anterior (drena para o seio cavernoso). A conexão dessas veias cerebrais é realizada pelas veias anastomóticas de Labbé e Trolard. Em relação ao seio sagital superior, é importante ressaltar que sua porção anterior pode não estar presente e, portanto, não se deve diagnosticar uma TVC erroneamente por sua ausência durante a avaliação por neuroimagem (ARAÚJO DS, 2017).

Estatisticamente, a TVC ocorre habitualmente nos seios venosos e, entre seios venosos e veias, sendo o seio sagital superior o mais afetado (cerca de $60-80 \%$ dos casos), seios transversos (41-42\%) e sigmoide, seios cavernoso e reto (18\%), seguido pelas veias corticais (17\%), seio cavernoso e as veias cerebelares. Já a oclusão da veia cerebral interna ocorre em 11\% dos casos (DUMAN T, et al., 2017; ARAÚJO DS, 2017).

Em cerca de dois terços dos casos ocorre TVC em mais de uma região. Neste contexto, há dois mecanismos que frequentemente ocorrem simultaneamente: a trombose de veias cerebrais, as quais conduzem turgência venosa e pode originar desde edema local a hemorragias petequiais e isquemia cerebral, podendo desencadear extensos hematomas e, por conseguinte, sintomas neurológicos focais, e; trombose de seios venosos durais, que leva a uma congestão venosa progressiva e redução da reabsorção do líquido 
cefalorraquidiano, ocasionando Hipertensão Intracraniana (HIC) a qual resulta em uma redução do aporte sanguíneo aos tecidos, causando hipóxia e isquemia neuronal (DUMAN T, et al., 2017; FERNANDES AMC, 2019).

Apesar de não ser completamente compreendida, a ocorrência da TVC está relacionada a desequilíbrios sistêmicos e locais, que envolvem processos trombóticos e trombolíticos que ocasionalmente podem levar à formação de trombos. A presença de trombos nos seios durais ou em qualquer veia cerebral pode ocasionar obstrução e, consequentemente, aumento da pressão venosa e redução da perfusão capilar (KRISTOFFERSEN ES, et al., 2018; BALIERO LG, et al., 2020).

A fisiopatologia do aumento da pressão intracraniana pode ser caracterizada pela presença de um trombo em qualquer sítio dos seios venosos cerebrais, os quais são responsáveis pela absorção do líquido cefalorraquidiano, assim, a não reabsorção ocasionada pela presença do trombo ocasiona a elevação da pressão. Nesse contexto, qualquer condição clínica que leve a alteração da capacidade de reabsorção dos seios venosos cerebrais, favorecendo o aumento da pressão intracraniana, pode também, predispor um episódio de trombose cerebral. Inicialmente, o aumento da pressão intracraniana é compensado pelo sistema colateral (ARAÚJO DS, 2017; SILVIS SM, et al., 2017).

Entretanto, com a evolução da doença, o aumento contínuo da pressão leva a quebra da barreira hematoencefálica e, consequentemente, extravasamento de líquido, ocasionando desde pequenas mudanças no parênquima cerebral até edema vasogênico localizado, infarto venoso e hemorragia. Dessa forma, quando o sistema colateral for suficiente para evitar o rompimento da barreira hematoencefálica, o quadro clínico do paciente estará mais relacionado à presença da hipertensão intracraniana, todavia, quando estes tornam-se ineficazes, ocorre congestão venosa e isquemia (SILVIS SM, et al., 2017).

Os principais fatores de riscos para a ocorrência de TVC podem ser divididos em duas categorias: permanentes e transitórios. Fatores de risco transitórios envolvem aqueles específicos do gênero feminino, tais como uso de contraceptivos orais, gravidez (principalmente no terceiro trimestre), puerpério (10 vezes maior durante as primeiras seis semanas após o parto), e aqueles não relacionados, envolvendo certos medicamentos, como quimioterapia, niveis de desidratação e algumas infecções, incluindo sinusite paranasal e mastoidite (ARAÚJO DS, 2017).

Dentre os fatores de risco permanente incluem estados protrombóticos hereditários, neoplasias, doenças inflamatórias sistêmicas, desordens hematológicas, doenças do SNC, tireoidopatia e distúrbios mieloproliferativos. Os estados protrombóticos, sejam eles congênitos ou adquiridos, e o uso de contraceptivos orais foram considerados fatores de risco mais importantes para predisposição de TVC. Cabese ressaltar que esta categorização continua sendo bastante superficial, colaborando com a definição multifatorial da TVC (ARAÚJO DS, 2017; GHONEIM A, et al., 2020).

Diante de um quadro de TVC, os sintomas podem variar de acordo com a extensão e local da doença, estes podem ser evidenciados na forma de síndromes, sendo as mais frequentes a síndrome de hipertensão intracraniana isolada, síndrome focal e encefalopatia, de forma menos frequente podemos observar, síndrome do seio cavernoso ou síndrome de paralisia múltipla dos nervos cranianos inferiores. Pacientes que desenvolvem a síndrome de hipertensão intracraniana isolada apresentam cefaleia, diminuição da acuidade visual e papiledema, para os que desenvolvem a síndrome focal, convulsões e déficits neurológicos podem estar presentes (FERRO JM, SOUSA DA, 2019; COUTINHO JM, 2015).

Dentre os sinais e sintomas descritos, a cefaleia é o sintoma mais comum, afetando cerca de $70 \%$ a $90 \%$ dos pacientes que pode ser fisiopatologicamente esclarecida pelo alongamento das fibras nervosas na parede do vaso, sucedido pela dilatação das veias do seio nasal e cortical espinhal, levando ao aumento da pressão intracraniana seguido de liberação de mediadores pró-inflamatórios podendo desencadear, por fim, uma hemorragia subaracnóidea (LV B, et al., 2021; MEHTA A, et al., 2019).

Tipicamente, a cefaleia pode ser localizada ou holocraniana. Pode inicialmente ser o único sintoma apresentado pelo paciente, entretanto, a maioria costuma evoluir também com sinais focais, tais como: déficits 
neurológicos, déficits motores e/ou convulsões. Contudo, pacientes idosos costumam ter a encefalopatia como manifestação mais frequente, apresentando confusão mental, perda de memória e convulsões, sendo a cefaleia mais comumente relatada em jovens (OLIVEIRA LF, et al., 2016; FERRO JM, SOUSA DA, 2019).

Ademais, diante das diferentes localizações anatômicas do trombo, podemos observar diferentes apresentações clínicas. Desse modo, trombose no seio venoso pode se apresentar com cefaleia, náuseas, edema papilar, diminuição da acuidade visual e alteração do nível de consciência, estes relacionados a hipertensão intracraniana. Trombos localizados em veias mais superficiais associados a lesões corticais/subcorticais, frequentemente podem apresentar convulsões e sintomas neurológicos focais. Além disso, trombos em veias mais profundas podem ocasionar edemas ao redor dos gânglios da base e do tálamo, ocasionando alteração do estado mental, distúrbios do movimento e, em casos mais graves, coma. Por fim, a presença de trombo no seio cavernoso está relacionada principalmente a neurite retrobulbar e oftalmoplegia (KRISTOFFERSEN ES, et al., 2018).

Apesar da alta porcentagem de pacientes que se recuperam da TVC, muitos apresentam sintomas crônicos residuais, cerca de $50 \%$ dos pacientes relataram cefaleia leve a grave durante o acompanhamento, levando uma pequena porcentagem para internação hospitalar. Prioritariamente, as diretrizes mais atuais já recomendam que pacientes com diagnóstico de TVC sejam amparados em unidades esspecializadas de AVE, disponibilizando assim, um suporte mais adequado para tal condição (SILVIS SM, et al., 2017).

Em casos de suspeita de TVC os exames de imagem possuem uma grande relevância na avaliação cerebral e vascular. Cada modalidade da neuroimagem possui uma vantagem distinta, sendo assim, investigações específicas devem ser adaptadas à suspeita clínica, levando em consideração as características individuais de cada paciente. Em primeira análise, o médico radiologista será o profissional responsável pela avaliação da imagem e diagnóstico, etapa de extrema importância para o seguimento do paciente (FERRO JM, SOUSA DA, 2019; WU X, et al., 2021).

São modalidades de neuroimagem disponíveis para o diagnóstico TVC: a TC, venografia por TC, RM, venografia por RM e angiografia por subtração digital. Nesse contexto, as potenciais complicações associadas à exposição à radiação e ao material de contraste intravenoso devem ser consideradas e a utilização destes métodos deve ser apurada minuciosamente em pacientes que requerem a investigação por neuroimagem. Contudo, apesar dos riscos, em casos de suspeita de TVC, os benefícios de um diagnóstico em tempo hábil e, por conseguinte, a implicação de métodos terapêuticos excedem os riscos (LV B, et al., 2021, LONG B, et al., 2017).

Usualmente, a tomografia computadorizada sem contraste é o primeiro método de investigação na sala de emergência por sua maior disponibilidade e rapidez, o exame ajuda a descartar situações que se confundam com a TVC, como neoplasia cerebral e hematoma subdural (OLIVEIRA LF, et al., 2016; WU X, et al., 2021; LV B, et al., 2021).

Na utilização da TC sem contraste, o sinal clássico de TVC aguda pode ser observado através do aumento da densidade dos seios. Além disso, podemos ter o sinal do delta vazio, caracterizando um coágulo no seio sagital superior, ou o sinal de cordão, caracterizando trombose na veia cortical. Entretanto, cabe ressaltar que esses sinais podem estar presentes apenas em uma parte dos pacientes e não são totalmente específicos da doença, sendo que $30 \%$ dos pacientes podem apresentar tomografia computadorizada (TC) normal no início dos sintomas. Portanto, uma TC de crânio sem contraste negativa não pode ser utilizada para descartar a doença (SILVIS SM, et al., 2017; LONG B, et al., 2017).

Apesar da TC ser uma grande aliada no diagnóstico, quando realizada sem contraste pode se apresentar de forma inespecífica e deixar dúvidas. Nesse contexto, a utilização do contraste deve ser instituída e alguns sinais indiretos de TVC podem ser notados, dentre eles, o derrame nos sulcos pode aparecer como uma das primeiras evidências de isquemia venosa, associado a sinais como edema parenquimatoso difuso, diminuição da diferenciação entre substância cinzenta e branca ou derrame ventricular. Outrossim, a presença de hemorragias justacorticais demonstraram ser bastante específicas para trombose do seio sagital superior (ARAÚJO DS, 2017; GHONEIM A, et al., 2020; SILVIS SM, et al., 2017). 
A TC pode ainda ser associada a venografia, método de imagem que fornece uma sensibilidade e especificidade entre $75 \%$ e $100 \%$, sendo determinada em relação ao local de ocorrência da trombose. A venografia por TC evidencia de forma detalhada o sistema nervoso cerebral, permitindo a correta identificação das veias cerebrais em $88 \%$ dos pacientes. Defeitos de enchimento no seio dural e nas veias, visto na venografia por TC, podem determinar o diagnóstico de TVC. Ademais, a venografia pode ser associada tanto a TC quando a RM, entretanto, a venografia por RM é mais sensível em detectar trombose das pequenas veias corticais com fluxo lento (GHONEIM A, et al., 2020; SILVIS SM, et al., 2017; FERRO JM e SOUSA DA, 2019).

Em relação à ressonância magnética, é a técnica mais amplamente usada para diagnóstico, devemos observar a presença do trombo dentro do vaso associado a ausência de fluxo, sendo este último observado na venografia por RM. Além disso, é aconselhado o uso de eco ou imagens ponderadas pela susceptibilidade T2 para otimizar o diagnóstico, sobretudo, na fase aguda da TVC e em pacientes com suspeita de trombose da veia cortical isolada. Durante a avaliação diagnóstica, nessas sequências, os trombos são identificados como áreas hipotensas, essas definidas pelo aumento dos níveis de desoxihemoglobina. Ressalta-se que, em relação a idade do trombo, o sinal apresentado na RM pode variar. Outrossim, durante a fase aguda da TVC, a venografia por RM, é ocasionalmente mais sensível em comparação a venografia por TC, entretanto, ambas podem confirmar o diagnóstico de TVC COUTINHO JM, 2015; SILVIS SM, et al., 2017; KRISTOFFERSEN ES, et al., 2018).

Caracteristicamente, dentro de 5 dias após o início do trombo, observa-se a presença de deoxihemoglobina em seu interior. Assim, o trombo se apresentará com isossinal em relação à substância cinzenta na imagem em T1 e com hipossinal na imagem em T2. O aparecimento da meta-hemoglobina a partir do sexto dia ao décimo quinto dia passa a ser evidenciado como hipersinal em T1 e T2. Nesse momento, na maioria dos casos o trombo aparecerá anormal, sendo permitido uma interpretação direta na RM. Ademais, $15 \%$ dos pacientes quando diagnosticados encontram-se com evolução acima de 15 dias da doença, sendo assim, o seio cronicamente trombosado, usualmente apresentará isossinal ou sinal hiperintenso em T2 e isointenso em T1 (GHONEIM A, et al., 2020).

A angiografia cerebral é considerada por algumas literaturas como "padrão ouro" para definição do diagnóstico definitivo de TVC. Entretanto, por ser um método invasivo e com riscos para acidente vascular cerebral, na maioria das vezes não será necessária, ficando reservada para pacientes hospitalizados que estão sendo avaliados para intervenções terapêuticas como trombólise ou implante de stent e, além disso, quando a TC ou RM são inconclusivas. A venografia por TC e a venografia por RM fornecem resultados análogos e, portanto, a escolha da técnica depende da situação clínica do paciente, experiência da equipe médica e recursos disponíveis. Ademais, a venografia por RM pode ser utilizada como uma alternativa à angiografia por subtração digital para a confirmação do diagnóstico em pacientes com suspeita de TVC (OLIVEIRA LF, et al., 2016; SILVIS SM, et al., 2017; WU X, et al., 2021; LV B, et al., 2021; FERRO JM, et al., 2017).

\section{CONSIDERAÇÕES FINAIS}

A TVC constitui uma doença com amplo espectro de manifestações, portanto, seu diagnóstico depende, sobretudo, da capacidade do profissional de reconhecer em tempo hábil suas possíveis síndromes clínicas e alterações parenquimatosas observadas por neuroimagem. Diante disso, a TC e a RM tornam-se pontos chaves para o diagnóstico definitivo. Assim, através do estudo das imagens é possível observar alterações secundárias à trombose, como hemorragia cerebral, edema e infarto. Outrossim, cabe destacar que, em comparação com a TC, a RM é considerada o melhor método para diagnóstico, podendo ainda ser associada a venografia, proporcionando uma melhor visualização das principais veias e seios cerebrais. Por fim, apesar de ser uma condição potencialmente grave, o diagnóstico rápido e acurado deve ser instituído a fim de garantir um melhor prognóstico a partir da implementação de um tratamento apropriado, reversão e controle de danos cerebrais e redução de complicações e sequelas. 


\section{REFERÊNCIAS}

1. ARAÚJO DS. Trombose venosa cerebral: desfecho cognitivo e avaliação clínico-radiológica de pacientes do Hospital Geral de Fortaleza. Dissertação (Monografia em Residência Médica em Neurologia) - Escola de Saúde Pública do Ceará. Hospital Geral de Fortaleza, 2017; 76 p.

2. BALIEIRO LG, et al. Trombose Venosa Cerebral: Aspectos Gerais e Métodos Diagnósticos. Brazilian Journal of Health Review, 2020; 3(1): 797-801.

3. COUTINHO JM. Cerebral venous thrombosis. Journal of thrombosis and haemostasis: JTH, 2015; 13(1): $238-244$.

4. DMYTRIW AA, et al. Cerebral venous thrombosis: state of the art diagnosis and management, 2018; 60(7): 669-685.

5. DUMAN T, et al. A Multicenter Study of 1144 Patients with Cerebral Venous Thrombosis: The VENOST Study. Journal of Stroke and Cerebrovascular Diseases, 2017; 26(8): 1848-57.

6. FERNANDES AMC. Trombose Venosa Cerebral: Revisão de Literatura. Dissertação (Mestrado em Medicina) - Centro Hospitalar Cova da Beira, Covilhã, 2019; 48 p.

7. FERRO JM, et al. European Stroke Organization guideline for the diagnosis and treatment of cerebral venous thrombosis. European Journal of Neurology, 2017; 24(10): 1203-1213.

8. FERRO JM, SOUSA DA. Cerebral Venous Thrombosis: an Update. Current Neurology and Neuroscience Reports, 2019; 19(10): 74.

9. GHONEIM A, et al. Imaging of cerebral venous thrombosis. Clinical Radiology, 2020; 75(4): 254-264.

10. HERNÁNDEZ JLM, et al. Trombosis venosa cerebral. Revista Cubana de Medicina, 2019; 58(2).

11. KRISTOFFERSEN ES, et al. Cerebral venous thrombosis - epidemiology, diagnosis and treatment. Tidsskr Nor Laegeforen, 2018; 138(12).

12. LONG B, et al. Cerebral Venous Thrombosis: A Challenging Neurologic Diagnosis. Emerg Med Clin North Am, 2017; 35(4): 869-878.

13. LV B, et al. Diffusion-Weighted Magnetic Resonance Imaging in the Diagnosis of Cerebral Venous Thrombosis: $A$ Meta-Analysis. Journal of Korean Neurosurgical Society, 2021; 64(3): 418-26.

14. MEHTA A, et al. Cerebral Venous Thrombosis Headache. Curr Pain Headache Rep, 2019; 23(7):47.

15. OLIVEIRA GHR, et al. Trombose Venosa Cerebral com Transformação Hemorrágica. Jornal Brasileiro de Neurocirurgia, 2018; 23(2): 346-349.

16. OLIVEIRA LF, et al. Trombose Venosa Cerebral e Alterações Cognitivas. Revista Brasileira de Neurologia e Psiquiatria, 2016; 20(1).

17. SILVIS SM, et al. Cerebral venous thrombosis. Nature Reviews, 2017; 13(9): 555-565.

18. WU X, et al. Magnetic Resonance Black-Blood Thrombus Imaging Can Confirm Chronic Cerebral Venous Thrombosis: A Case Report and Literature Review. Journal of International Medical Research, 2021; 49(5): 03000605211017001.

19. ZEDES GD, et al. Trombose Venosa Cerebral - Causas, Sintomas e Tratamentos. Revista Liberum Accessum, 2020; $4(1): 38-45$.

20. ZUURBIER SM, et al. Cerebral Venous Thrombosis in Older Patients. Stroke, 2018; 49(1): 197-200. 